\title{
Hybrids of the Hemiasterlin Analog Taltobulin (HTI-286) and the Dolastatins are Potent Antimicrotubule Agents
}

Arie Zask, ${ }^{* 1}$ Joshua Kaplan, ${ }^{1}$ Sylvia Musto, ${ }^{2}$ Frank Loganzo ${ }^{2}$

${ }^{1}$ Chemical and Screening Sciences, ${ }^{2}$ Oncology Research, Wyeth Research, 401 North Middletown Road, Pearl River, NY 10965 USA

\section{Supporting Information}

\section{References}

${ }^{8}$ Loganzo, F.; Discafani, C.M.; Annable, T.; Beyer, C.; Musto, S.; Hari, M.; Tan, X.; Hardy, C.; Hernandez, R.; Baxter, M.; Singanallore, T.; Khafizova, G.; Poruchynsky, M. S.; Fojo, T.;

Nieman, J. A.; Ayral-Kaloustian, S.; Zask, A.; Andersen, R. J.; Greenberger, L. M. Cancer Res. 2003, 63, $1838-1845$.

${ }^{9}$ Zask, A.; Birnberg, G.; Cheung, K.; Kaplan, J.; Niu, C.; Norton, E.; Suayan, R.; Yamashita, A.; Cole, D.; Tang, Z.; Krishnamurthy, G.; Williamson, R.; Khafizova, G.; Musto, S.; Hernandez, R.; Annable, T.; Yang, X.; Discafani, C.; Beyer, C.; Greenberger, L.M.; Loganzo, F.; AyralKaloustian, S. J. Med. Chem. 2004, 47, 4774-4786.

${ }^{14}$ Zask, A.; Birnberg, G.; Cheung, K.; Kaplan, J.; Niu, C.; Norton, E.; Yamashita,; A. Beyer, C.; Krishnamurthy, G.; Greenberger, L.M.; Loganzo, F.; Ayral-Kaloustian, S. Bioorg. Med. Chem. Lett. 2004, 14, 4353-4358. 